REVISTA DE INVESTIGACIÓN

EN GESTIÓN CULTURAL

Córima, Revista de Investigación en Gestión Cultural

ISSN electrónico: 2448-7694

Universidad de Guadalajara

Sistema de Universidad Virtual

México

corima@udgvirtual.udg.mx

Año 4, número 7, julio-diciembre 2019

\title{
El creador emergente como gestor, proveedor y administrador de servicios culturales en instituciones gubernamentales. El caso del Ayuntamiento de Guadalajara, México
}

\author{
Pedro Ramírez Hernández ${ }^{1}$ \\ Centro Universitario UTEG, México
}

DOI: $10.32870 /$ cor.a4n7.7339

[Recibido: 16/01/2019; aceptado para su publicación: 10/06/2019]

\section{Resumen}

Vivir de la cultura es un reto, y para lograrlo es necesario diversificar las fuentes de ingresos. Una de ellas es la oferta de servicios culturales que un creador emergente puede realizar como proveedor a clientes interesados en su adquisición, en este caso, instituciones gubernamentales en materia de cultura. Sin embargo, esta lucrativa dinámica implica muchos obstáculos. Este artículo tiene como propósito presentar las dificultades que enfrenta el creador emergente en el proceso de participación, como proveedor de servicios y productos culturales en las instituciones gubernamentales responsables de las políticas culturales en el

\footnotetext{
${ }^{1}$ Correo electrónico: pedro.culturagdl@gmail.com
}

CÓMO CITAR ESTE ARTÍCULO:

Ramírez Hernández, P. (2019). El creador emergente como gestor, proveedor y administrador de servicios culturales en instituciones gubernamentales. Caso del Ayuntamiento de Guadalajara. Córima, Revista de Investigación en Gestión Cultural, 4(7). doi: 10.32870/cor.a4n7.7339 
Estado de Jalisco, México. Se analizó el caso del Ayuntamiento de Guadalajara, el cual, para la adquisición de servicios, debe seguir la normatividad que marca la autoridad estatal. Se realizó un profundo análisis del proceso de proveeduría de productos culturales, entrevistas con funcionarios responsables de la ejecución de recursos, perspectivas de creadores emergentes interesados en ser proveedores del municipio. Por último, percepciones de creadores que ya son proveedores. Del análisis de lo anterior se permite tener un panorama de las barreras que elementos como, la burocracia y la propia comunidad cultural han generado en perjuicio del creador emergente.

\section{Palabras clave}

Políticas culturales, capacitación de la gestión cultural, emprendimientos culturales.

\section{Introducción}

Se parte de la premisa de que un creador emergente es aquel que, independientemente de su edad, se encuentra en una etapa inicial de su quehacer creativo, y que busca consolidar su posicionamiento como profesional a través de gestiones y medios propios (Almela, 1998). Dicha capacidad de autogestión cobra mayor relevancia si se relaciona con las cualidades que un creador y estudiante de programas de educación superior requiere para insertarse eficientemente en el campo laboral; especialmente, si sus inquietudes lo encaminan a autoemplearse en su propio negocio ya sea dentro de su actividad creativa, en áreas de gestión cultural o fungir como asesor especializado en los aspectos de la cultura.

Sin embargo, en el ámbito profesional es común encontrar situaciones en donde la obtención de ingresos económicos, producto de actividades como creador (especialmente en el ramo cultural), se les suelen vincular con el acceso a programas de mecenazgo (principalmente gubernamentales), como patrocinios, premios, becas o subsidios, cuyo acceso está limitado (debido a que estos recursos tienen un tope presupuestal) a unos cuantos seleccionados, que reúnen una serie de criterios de calidad y pertinencia, los cuales son dictaminados y percibidos por un jurado compuesto de expertos en determinadas áreas (Corona, 2017).

A su vez, los montos de estos recursos no siempre son lucrativos para el creador, y debido a que están sujetos a diversas temporalidades no se pueden considerar como una fuente estable de ingresos. De esto nos habla un creador emergente al mencionar que en "México existe la tradición de vivir del Estado y hasta ahora las únicas posibilidades de prestigio son las becas [...], las que no son para nada jugosas" (Carmona, 2015). 
La búsqueda por acceder a este tipo de ingresos relacionados con el mecenazgo ha provocado que existan actividades de adiestramiento, como la impartición de cursos y talleres; un ejemplo de ello es el Simposio Internacional de Arte, Cultura y Negocios, que en palabras de su codirector Sergio Gómez-Tagle (2017), uno de los ejes principales es el de "instruir a los promotores culturales en los gajes del financiamiento y patrocino de las artes". Si bien este mismo autor manifiesta la importancia de diversificar las alternativas de ingresos ante la falta de fondos suficientes por parte del Estado, se refiere a estas alternativas como "patrocinios, alianzas estratégicas y estímulos fiscales" (Gómez-Tagle, 2017), los cuales pueden considerarse dentro de las actividades de mecenazgo.

En un contexto local encontramos actividades educativas como las propuestas por instituciones como la Secretaría de Cultura del Gobierno del Estado de Jalisco (2018), que por una parte buscan el fortalecimiento de un sector empresarial orientado a creadores, y por otro, dentro de sus ejes de trabajo se pueden vislumbrar similares estrategias de mecenazgo y apoyos de financiamiento, como el programa Proyecta Industrias Creativas, el cual basa su aplicación de recursos en criterios de selección a cierto número limitado de seleccionados bajo procesos similares a los antes planteados.

Ante tal panorama es lógico pensar que las estrategias de ingresos económicos, cuyo origen es producto de estos modelos, no son parte de estrategias de subsistencia a largo plazo. A su vez, al tomar en cuenta el sentido ideal de las actividades empresariales, en donde los participantes deben tener acceso a beneficios y lucrativos ingresos, los cuales deben estar alineados con los intereses de todos los involucrados (Bossi, 2015), las prácticas derivadas del mecenazgo contrastan con este precepto, ya que por lo general buscan el beneficio del receptor, basándose principalmente por su naturaleza de "ayuda dispensada" (RAE, 2018).

Derivado de la reflexión anterior, uno de los posibles escenarios que el creador emergente tiene para lograr estabilidad profesional a largo plazo, es formalizar y operar un negocio propio, el cual, si contempla líneas de trabajo como la innovación constante y una administración responsable, podrá obtener posicionamiento comercial.

Este último punto en relación con una administración y operación responsable de un proyecto de negocio, es un tópico con el que comúnmente no se relaciona al creador (principalmente en su etapa como estudiante o recién egresado de algún programa de educación superior), el cual, en el imaginario colectivo, se le vincula con la informalidad laboral. Sin embargo, es importante mencionar que en la actualidad existen sectores creativos que han mostrado avances trascendentales en la búsqueda 
de formalizar su actividad comercial; un ejemplo de ello son los artesanos jaliscienses, quienes con mayor frecuencia buscan constituirse como empresa (esto reporta el Director del Instituto de la Artesanía Jalisciense, Ernesto Meza Tejeda, el cual menciona que en 2017 se constituyeron 16 nuevas cooperativas y se registraron 31 marcas del sector [Meza, 2018]).

Naturalmente, un negocio necesita clientes; en este caso, que estén interesados en invertir recursos para la adquisición de productos o servicios culturales. Esto ha provocado que el creador busque este tipo de relaciones, en especial con la iniciativa privada, en donde las actividades se concentran en transacciones de compraventa minorista de productos artísticos.

Sin embargo, al concentrar el ingreso económico en una sola actividad comercial (venta al detalle), la existencia de competencia desleal aumenta y pone en peligro la estabilidad laboral. Ante esta circunstancia, un creador profesional, apoyado en su creatividad, debe poseer la capacidad de detectar problemas y proponer soluciones ante este escenario, derivado de una "fuerte vinculación con su entorno social, siendo capaz de reflexionar sobre él" (LAV, CUAAD, 2019). Frente a esta situación, una de las soluciones recomendadas para un creador y estudiante de educación superior es diversificar sus fuentes de ingreso (Gómez-Tagle, 2017).

Ante estos esquemas comerciales, las actuales estrategias de marketing, plantean que es necesario buscar nuevas alternativas que signifiquen un contrapeso ante las teorías tradicionales de mercadeo, alternativas que incluyan la concepción y desarrollo de instrumentos y estrategias propias del creador, las cuales a la par de ser producto de su creatividad, deben estar adaptadas a las necesidades del entorno cultural. Para que esto sea eficaz, naturalmente el creador debe ser un observador meticuloso de su realidad.

En la lectura contextual que el creador forme de su entorno, se debe tener en cuenta que los productos y servicios cultures se ven fortalecidos cuando mantienen relación con múltiples grupos de interés (Leal \& Quero, 2013). Es decir, el marketing cultural se desarrolla entre las organizaciones culturales y en la sociedad para crear intercambios de colaboración recíproca.

Naturalmente para que una colaboración sea fuerte se deben buscar alianzas con entidades que tengan los mismos fines, es decir, que estén interesadas en beneficiarse con la producción cultural que un creador puede ofertar. Por tal motivo, antes de buscar una colaboración, se deben conocer los beneficios que puede aportar una propuesta, y si estas aportaciones presentan innovadoras formas de comercialización. Para clarificar esto, la gestora cultural María José Quero aconseja que el creador se realice tres cuestionamientos: ¿cuáles son los principios que un 
cliente puede valorar en una oferta?, ¿qué clientes buscan beneficios que se puedan alinear con determinada oferta? y ¿cuáles son las prioridades de los clientes con quien se quiere colaborar? (Manual de Marketing y Comunicación Cultural, 2013).

Con este tipo de preguntas, el diseño de nuevas estrategias de captación de ingresos puede estar apoyado de un plan estratégico flexible y adaptable a las circunstancias que se presenten.

\section{La adquisición de servicios culturales por parte del Ayuntamiento de Guadalajara}

Las recomendaciones que invitan a forjar relaciones colaborativas de mutuo beneficio, y bajo la premisa que en el sector de la gestión cultural y patrimonial la obtención de ingresos económicos no solo se basa en estrategias de compraventa, sino que actividades como la difusión, el resguardo, el fomento, el rescate y la difusión pueden generar productos susceptibles de obtener ingresos, se sugiere detectar a los clientes que necesiten de estos productos para beneficiarse con ellos.

En este sentido, destacan las entidades gubernamentales, quienes por normatividad deben disponer recursos para la difusión y protección cultural; este es el caso del Ayuntamiento de Guadalajara el cual, entre sus múltiples responsabilidades, debe:

I) Realizar los proyectos, programas y mecanismos, que impulsen y fomenten el desarrollo de las actividades culturales y artísticas que se desarrollen en el Municipio de Guadalajara, para hacer vigente una cultura de paz.

$[\ldots]$

XX) Coadyuvar en la preservación del patrimonio cultural y artístico del municipio;

XXI) Promover la difusión del patrimonio y tradiciones culturales y artísticas del municipio (Reglamento de la Administración Pública Municipal de Guadalajara, 2018, artículo 144).

Si bien las actividades de estímulo y mecenazgo que implementa el Ayuntamiento de Guadalajara dan cumplimiento parcial a estas obligaciones, los recursos gubernamentales destinados para la operación de estos programas (bajo el esquema de subsidios etiquetados) son mínimos (aproximadamente \$2 000 000.00); en comparación a lo dispuesto para la adquisición de productos o servicios que dan cumplimiento total a sus obligaciones culturales, los cuales en el ejercicio fiscal de 
2018 fueron de \$58 059 702.00, tan solo para la Dirección de Cultura (Gaceta Municipal del Ayuntamiento de Guadalajara, 2017).

A su vez, la ejecución y acceso a estos recursos se encuentran dirigidos a un esquema de proyectos que están destinados a cumplir los objetivos específicos, concentrados en alcanzar la eficacia de la dependencia en torno a la normatividad que la atañe y las metas institucionales en turno:

\section{Tabla 1}

\begin{tabular}{|c|c|}
\hline Proyecto & Monto \\
\hline Cultura en tu comunidad & $\$ 6,574,881.00$ \\
\hline
\end{tabular}

Fuente: Presupuesto de Egresos del Municipio de Guadalajara, ejercicio fiscal de 2018.

En relación con los proyectos de índole cultural, es comprensible pensar que las dependencias de gobierno, por su nivel de injerencia, son capaces de llevar a cabo actividades culturales para cumplir los proyectos estipulados; sin embargo, derivado de diversas circunstancias (como un proceso de reclutamiento de personal que no se basa en la búsqueda de perfiles ideales para el desempeño de diversos puestos, situación que en parte es originada por el hecho de que para la colocación de personal "el gobierno en turno politiza los cargos y puestos, lo que puede derivar en casos de corrupción" [Aziz, 2017], así como limitados programas de capacitación que incentiven y certifiquen al personal), se origina un contexto en donde las instituciones tienen una carencia de personal calificado para el desarrollo de sus actividades. Por este motivo las dependencias se encuentran limitadas para cumplir la totalidad de sus actividades (Ramírez, 2016), lo que ocasiona que para la ejecución de sus proyectos, los organismos gubernamentales dependan en gran medida de la adquisición de servicios culturales proporcionados por agentes externos, es decir, proveedores.

Entre los servicios que son susceptibles de adquirir por parte del Ayuntamiento de Guadalajara, se encuentran:

Tabla 2

\begin{tabular}{|l|l|}
\hline Eventos de giro cultural y patrimonial & Talleres artísticos \\
\hline Investigación & Avalúos \\
\hline Festivales culturales y patrimoniales & Revisión y dictamen de colecciones \\
\hline Exposiciones (curaduría y producción) & $\begin{array}{l}\text { Programas de rescate y protección } \\
\text { patrimonial }\end{array}$ \\
\hline Talleres culturales o artesanales & Restauración y conservación \\
\hline
\end{tabular}


Renovación y rehabilitación de espacios culturales
Programas de capacitación cultural

Fuente: 3er Informe de Gobierno, Ayuntamiento de Guadalajara, 2018.

Es importante mencionar que estos servicios pueden ser proporcionados plenamente por un profesional que se dedique a la creación o gestión cultural. Sin embargo, se debe tener en cuenta que en el caso del Ayuntamiento de Guadalajara, para participar en el suministro de servicios se debe tener la figura de proveedor, el cual está definido como "persona física y jurídica que se registre ante la entidad, y tenga la finalidad de proveer los bienes o servicios que requiera el municipio" (Reglamento de Adquisiciones para el Municipio de Guadalajara, 2006, artículo 61).

Esta dinámica de adquisición de servicios culturales por entidades gubernamentales que requieren satisfacer necesidades por normatividad u objetivos institucionales, representa una alternativa importante para el creador que desea diversificar sus fuentes de ingresos. El suministro de servicios culturales significa una oportunidad para acceder a recursos gubernamentales que no están sujetos a actividades de mecenazgo y que a su vez, no son parte de transacciones comerciales de compraventa de artículos o artesanías. Sin embargo, la participación en este lucrativo esquema requiere una serie de conocimientos en materia de gestión administrativa y habilidades (como la constancia), con las que no cuentan todos los creadores, o que simplemente no conciben como necesarias para la diversificación de fuentes de ingresos (Ramírez, 2016).

\section{Metodología}

Este trabajo comenzó con un análisis detallado del proceso de adjudicación y compra en el Ayuntamiento de Guadalajara en materia de cultura. De las diversas variantes para adquirir bienes o servicios, se detectó el mecanismo utilizado con más frecuencia para ese tipo de compras, lo que permitió identificar a los proveedores recurrentes que participan en este proceso, así como las características que poseen, la relación que entablan con las dependencias y sus percepciones particulares en torno a esta dinámica de compra-venta.

Las compras analizadas fueron las relacionadas con la adquisición de exposiciones y servicios de los seis museos con los que cuenta la Dirección de Cultura del Ayuntamiento de Guadalajara, durante el período 2016-2018. Dicha información fue clasificada por espacio, recurrencia de los proveedores, funcionario solicitante, características del servicio adquirido y monto de la compra. Cabe mencionar que el 
95\% de las adquisiciones en exposiciones fueron bajo un esquema de "adjudicación directa" (sin licitación), el cual se describe más adelante.

Dicha información se recopiló mediante los mecanismos de transparencia del municipio, cotejo documental de órdenes de compra, así como seguimiento de la agenda de eventos culturales para analizar las características de lo adquirido por el municipio. A su vez, se realizaron entrevistas con funcionarios públicos de la Dirección de Cultura responsables de la ejecución de recursos para el cumplimiento de determinados proyectos.

Por último, se detectó un grupo de creadores emergentes que eran son proveedores del Ayuntamiento, y que estuvieran interesados en serlo, para recabar sus inquietudes y experimentar junto con ellos todo el proceso para registrarse como proveedores; estas percepciones fueron recabadas a través de creadores que participaron en proyectos como actividades del Museo Panteón de Belén, Galerías Urbanas y el proyecto "Arte Público".

\section{La problemática de los creadores culturales y su inserción en el proceso de proveeduría del Ayuntamiento de Guadalajara}

Es innegable el hecho de que cuando se habla de inversión financiera en materia de cultura, el Estado destaca por ser un cliente altamente atractivo, principalmente si se desea emprender en el giro de servicios culturales. Sin embargo, para establecer una relación comercial con él, se requiere por parte del creador una serie de características, formalidades y requisitos (los cuales en primera instancia pueden parecer numerosos), que en muchas ocasiones desconoce. De esto nos habla Sergio Gómez-Tagle, quien comenta que "el panorama de las empresas creativas en México está creciendo, pero aún no se tiene conciencia de la tramitología y de los procesos administrativos que deben llevarse a cabo" (2017).

Lo anterior cobra mayor relevancia una vez que se traslada este escenario al proceso de adquisiciones de servicios culturales. En la información obtenida del ex coordinador de Museos y Galerías (2016-2018), maestro Pedro Ramírez, se manifiesta que al revisar la base de datos de proveedores pertenecientes a giros culturales, con los que se pueda adquirir servicios como producción de exposiciones, la lista es escasa, por lo que se obstaculiza implementar dinámicas de rotación en la adquisición de servicios (Ramírez, 2016).

El municipio es responsable de ofertar contenidos culturales a la ciudadanía, lo que sumado al reducido número de proveedores y escasez de personal calificado para 
la producción de actividades culturales, se origina una dinámica en la que se opta por dos caminos:

1) Negociar con alguno de los proveedores ya registrados, que conocen la burocracia institucional y que tienen un tiempo considerable proveyendo servicios culturales, para que subcontrate o represente a un creador cuyos servicios son de interés para el municipio, pero que por diversas circunstancias no puede darse de alta como proveedor (principalmente porque desconoce $\mathrm{o}$ no cuenta con los documentos formales para registrarse). Esta opción representa una temporalidad menor en temas logísticos.

2) Al detectar un servicio cultural de interés, se contacta al creador para invitarlo y convencerlo de que se registre como proveedor del municipio. En este escenario es común que el funcionario público acompañe con un asesoramiento y seguimiento constante al creador, para que su registro sea completado de manera satisfactoria. Esta opción tarda más tiempo en llevarse a cabo debido a los plazos estipulados por el trámite de registro y en muchas ocasiones impide al municipio adquirir servicios a corto plazo.

Este segundo punto presenta complicaciones mayores para un creador emergente, debido a que en el momento en que se le invita a ser parte del listado de proveedores, derivado de que se detectó que su producción es pertinente para la dependencia:

Es común encontrar situaciones en las que el creador emergente no cuenta con la información necesaria sobre la importancia de reunir ciertas características indispensables de formalidad administrativa, como la posibilidad de facturar debido a un correcto registro ante la Secretaria de Hacienda y Crédito Público, o la redacción, desglose y presentación de una cotización formal, por mencionar algunas. Requerimientos que son necesarios para materializar la ejecución de recursos públicos a través de la compra-venta de sus servicios (Ramírez, 2016).

Esto último se refuerza al recabar las percepciones de los creadores emergentes interesados en este esquema de ingresos, quienes en su mayoría manifestaron no estar actualizados en relación con los requerimientos administrativos que se necesitan para autoemplearse de esta manera. A su vez, cuando se les consultó el porqué de esta situación, mencionaron que su actividad profesional se concentraba en la creación, y en el caso de creadores emergentes que son egresados de esquemas de educación superior, en su mayoría expresaron que en el transcurso de su formación 
académica no se estudian asignaturas de gestión administrativa entorno a la creatividad.

Cada escenario presenta sus complicaciones y vicios ocultos. Si bien el negociar con un proveedor ya registrado para subcontratar a otro proveedor sin registro es una opción que permite resolver a corto plazo la adquisición de servicios culturales, esto ha provocado que los proveedores vigentes concentren sus esfuerzos en fortalecer una imagen comercial como gestores o representes cuyo principal aporte es la resolución de "problemas" administrativos, tanto para creadores como para dependencias culturales. Naturalmente, el éxito comercial de esta estrategia depende de factores como:

a) Una limitada oferta de proveedores de servicios culturales registrados.

b) El desconocimiento por parte de otros creadores emergentes sobre del proceso de registro como proveedor en materia cultural.

c) La apatía de los creadores en participar en dichos procesos administrativo.

Un dato de vital importancia es que dentro de los procesos de adquisición por parte del Ayuntamiento de Guadalajara, existen mecanismos para evitar prácticas que beneficien a un solo proveedor, como los concursos libres o licitaciones públicas, los cuales tienen la finalidad de que todos los proveedores de un giro particular puedan presentar sus propuestas y concursar por una designación para suministrar al ayuntamiento algún producto o servicio. Sin embargo, también se contempla la opción de asignar una compra de manera directa a algún proveedor, sin necesidad de un concurso. Esto sucede cuando se reúnen ciertas características:

Cuando resulte imposible la celebración de concursos debido a que no existan suficientes proveedores o se requiera de un bien con características o patente propia vigente, otorgada por la autoridad competente en México, así como aquellos con derechos protegidos de propiedad intelectual, previa justificación por parte de quien lo solicite (Ley de Adquisiciones y Enajenaciones del Estado de Jalisco, 2015, art. 14, inciso I).

Lo anterior cobra gran relevancia porque al no existir especificaciones sobre lo que se considera un servicio cultural en el clasificador de objetos de gasto de la Ley de Adquisiciones, ni en los reglamentos municipales, provoca que, apoyados en la ambigüedad del artículo e inciso antes citado y bajo el precepto de que "el autor es el único, primigenio y perpetuo titular de los derechos morales sobre las obras de su creación" (Ley Federal del Derecho de Autor, 1996, artículo 18), los proveedores de servicios culturales registrados etiqueten los productos o servicios que ofertan, como 
de bienes de características propias; lo que permite que sean adquiridos bajo el esquema de adjudicación directa, evitando los concursos públicos y garantizando que el proveedor sea el único oferente para esa compra.

En pocas palabras, si una dependencia gubernamental está interesada por el servicio cultural que determinado proveedor oferta, su adquisición será bajo una asignación de compra directa, situación que se presenta en la mayoría de las compras de índole cultural del Ayuntamiento de Guadalajara (80\%).

Para que un proveedor se desenvuelva constante y exitosamente en el lucrativo contexto descrito, y pueda considerar esta fuente de ingresos como fija, se necesitan dos factores:

1) La pericia y experiencia del proveedor para conocer plenamente el esquema de compras, así como estar alerta de las dificultades que las dependencias gubernamentales enfrentan para la ejecución de sus propios recursos públicos en materia de cultura.

2) La poca existencia proveedores de servicios culturales, registrados ante el Ayuntamiento de Guadalajara, para fortalecer su imagen de proveedor único.

A lo anterior se suma la preferencia que ciertas dependencias gubernamentales han mostrado por trabajar con proveedores con quienes han tenido experiencias anteriores (principalmente porque les representan comodidad y resolución a dificultades administrativas) y que los esfuerzos por incrementar el número de proveedores de servicios culturales son practicantes nulos. Esto afecta principalmente al creador emergente, el cual al encontrarse en una etapa inicial en su quehacer creativo, necesita diversificar sus fuentes de ingresos (con clientes de alto perfil), y justamente los servicios culturales son eso, un agente diversificador de ingresos en relación con la creatividad.

\section{¿Por qué es importante que un creador emergente diversifique sus fuentes de ingresos como proveedor de servicios culturales en el Ayuntamiento de Guadalajara?}

El fortalecimiento de la participación del creador emergente como proveedor de productos y servicios culturales es de vital importancia para su desarrollo profesional, ya que esto le permite ampliar el espectro de posibilidades para diversificar las fuentes de ingresos relacionadas con su actividad creativa. 
En el caso de que un creador emergente conozca y ejercite el ofrecimiento de servicios culturales, susceptibles de ser adquiridos por el ayuntamiento en cuestión, a la par se estará preparando para participar y repetir esta actividad de diversificación en distintos ayuntamientos y dependencias en todo el estado de Jalisco, debido a que todos llevan procesos de adquisición similares al del municipio de Guadalajara.

Si bien cada uno tiene su reglamento y proceso interno, este se encuentra sometido a los lineamientos jurídicos y operativos que ordena seguir la Ley Estatal de Adquisiciones y Enajenaciones del Estado de la Jalisco. A su vez, el utilizar como eje de aprendizaje el esquema de adquisiciones de este ayuntamiento, le permite al creador capacitarse en procesos complejos como lo es el del municipio que más recursos ejerce en materia de productos y servicios culturales.

En el caso de las instituciones gubernamentales, una mayor oferta de proveedores de servicios culturales le permitiría incrementar sus opciones para ofrecer actividades de cultura que por ley deben proporcionar a la ciudadanía, y de esta manera se les "libera" de recurrir a la práctica constante de trabajar con los proveedores conocidos por proporcionar "practicidad logística" dentro de la entramada burocracia interna. A su vez este ejercicio presiona colateralmente a los proveedores registrados a participar en una dinámica de mayor competencia.

Sin embargo, al analizar el perfil profesional del creador emergente (principalmente si es recién egresado de algún programa académico superior), este no posee la suficiente información o herramientas complementarias de gestión administrativa, que le permitan estar capacitado con los elementos para participar eficientemente en el esquema de adquisición de servicios culturales por parte del Ayuntamiento de Guadalajara, o con cualquier cliente de alto perfil.

En relación con lo anterior, en diversos programas académicos orientados a la práctica artística o creativa, se manifiesta que es importante un perfil de egreso compuesto, entre otras cosas, por características que le permitan posicionarse de diversas maneras en el mercado empresarial, así como desenvolverse en un ámbito laboral diversificado (LIDA, Perfil de egreso, 2018). Sin embargo, al tener presente la realidad que se vive en ambientes de competencia como el descrito en este artículo, las instituciones educativas pueden entablar una constante y enriquecedora discusión en torno a detectar la pertinencia de sus planes educativos en este aspecto, así como analizar si el diseño curricular actual prepara al estudiante con las herramientas de gestión administrativa necesarias para el pleno desarrollo en estos ambientes.

De materializar la discusión curricular mencionada, es posible que la línea de investigación de este artículo tome una directriz de implicaciones prácticas, situación que autores como Ackoff (1973) y Miller y Salkind (2002) consideran pertinentes para 
manifestar la necesidad de un proyecto de investigación (citado en Hernández, Fernández, \& Baptista, 2014, p. 40).

\section{Conclusiones}

Como ya se mencionó, el proveer servicios culturales al Ayuntamiento de Guadalajara significa participar en un lucrativo esquema de ingresos económicos en torno a la creatividad, en el que si el creador materializa administrativamente un perfil profesional formal, podrá diversificar la fuente de ingresos, sin necesidad de ejercitar el tradicional proceso de compra-venta de obra creativa.

De formalizar la participación en el Ayuntamiento de Guadalajara, se tendrá conocimiento para ingresar como proveedor en todas las dependencias del Estado de Jalisco, lo cual significa una cartera importante de clientes. Sin embargo, en el caso del creador emergente, este se enfrenta a múltiples obstáculos para entrar en esta dinámica, derivados de cada uno de los sectores involucrados:

1) Los proveedores que actualmente están registrados como entes que pueden suministrar servicios culturales en el Ayuntamiento de Guadalajara, para minimizar la competencia, emplean prácticas cuyo propósito es obstaculizar el aumento de proveedores en materia cultural en el Municipio de Guadalajara. Esta práctica se potencializa al fomentar el desinterés y la apatía de los creadores emergentes con argumentos como la complejidad de los procesos, producto de una burocracia "caótica". Es importante mencionar que para el cumplimiento de los servicios culturales ofertados, los proveedores registrados subcontratan o representan a creadores emergentes que no pueden o desconocen cómo ser proveedores. De esta manera, cumplen con lo acordado y obtienen ingresos constantes por la "resolución" administrativa y documental que proporcionan tanto para la dependencia como para el creador. Sin embargo, el creador emergente (quien es realmente el creador y proveedor del producto o servicio cultural) solo recibe una parte de los recursos ejecutados.

2) En materia de cultura, el Ayuntamiento de Guadalajara tiene inclinación por trabajar con proveedores con los que ya ha tenido experiencias anteriores, principalmente porque le significa certeza en relación con la forma de trabajar y experiencia del proveedor para desenvolverse en el sistema de adquisiciones del ayuntamiento. Uno de los principales motivos es la duración de la administración gubernamental, la cual tiene una temporalidad de tres años, tiempo que se considera limitado si el gobierno 
en turno desea evidenciar resultados a corto plazo. Si a lo anterior se agrega que el registro de proveedores nuevos y la adaptación que le significa a la dependencia el trabajar con proveedores con los que no ha tenido experiencias previas le implica mucho más tiempo para la ejecución de estrategias y políticas públicas, en comparación al trabajo con proveedores con los que ya ha tenido relación, es comprensible que se dé el escenario en donde el ayuntamiento prefiera los esquemas conocidos, aunque esto signifique que de manera colateral no incentive el registro ni participación de nuevos proveedores, principalmente si se trata de creadores emergentes que se encuentran en la consolidación de su inserción en el mercado laboral cultural.

3) Cuando un creador formaliza su figura como proveedor de servicios culturales, le proporciona ventajas competitivas significativas en comparación con profesionales que solo se dedican a una actividad, ya sea la gestión o la creación. Estas ventajas se pueden desglosar en los siguientes rubros:

- Relacionado con los procesos institucionales, el ser creador y proveedor le permite que toda la documentación y la comprobación administrativa de los gastos estén bajo su supervisión y autorización, lo que proporciona a la institución un mayor grado de certeza y confianza que se refleja en una mayor fluidez burocrática, debido a que existen menos involucrados.

- Al ser el autor de la propuesta o servicio cultural tiene la posibilidad de tomar decisiones inmediatas para flexibilizar o adaptar sus ofertas en torno a las necesidades o situaciones imprevistas de la institución, y que requieren solución.

- El creador posee conocimiento de primera mano en torno a las filosofías, costumbres o percepciones laborales del gremio creativo.

- La figura de creador-gestor-proveedor lo libera de la utilización de agentes externos para la difusión o comercialización de su actividad creativa; es decir, elimina los intermediarios administrativos entre el cliente y el productor, magnificando la captación de ingresos en su beneficio.

- Le permite al creador la posibilidad de colocarse como un referente que pueda ser guía y apoyo para otros creadores emergentes. 
4) En el momento que un creador emergente decide optar por la proveeduría de servicios culturales a potenciales clientes, es común que el creador se enfrente a un panorama en donde las características y requerimientos administrativos con los que debe contar para materializar su objetivo, le sean en su mayoría desconocidos. Principalmente porque no cuenta con los conocimientos necesarios ni las herramientas que requiere para desenvolverse como profesional en la gestión de servicios culturales. En el caso de creadores emergentes egresados de sistemas de educación superior, esto es derivado del plan de estudios que cursaron, en el cual no se estudiaron a profundidad los temas de gestión administrativa y su relevancia en la competencia laboral.

Esto último se considera que es la piedra angular para diseminar paulatinamente las dificultades que enfrenta el creador emergente en su inmersión en la dinámica laboral descrita en este documento.

Si bien las instituciones académicas enfocadas a la creación orientan sus esfuerzos en el desarrollo de las capacidades creativas que le permitan al creador destacar rápidamente en un mundo de intensa competencia. También es necesario capacitar al creador emergente para participar en los procesos administrativos de gestión que le permitan diversificar y ofertar sus productos y servicios culturales con clientes de alto perfil adquisitivo.

Para ello se debe entablar un necesario, responsable y respetuoso análisis en relación con la pertinencia del mapa curricular de los sistemas de educación, para encontrar si las asignaturas y objetivos actuales están otorgándole al estudiante las características antes mencionadas.

En la dinámica laboral actual es necesario fortalecer esta línea de aprendizaje, para que, a través del conocimiento de temas administrativos y legislativos orientados a la creatividad, el creador consolide un perfil profesional preparado para un ámbito laboral diversificado.

Solo un creador emergente, dotado de una educación integral compuesta de habilidades creativas y administrativas, podrá participar en escenarios lucrativos como ser proveedor de servicios culturales en el Ayuntamiento de Guadalajara. De esta manera ejercitará eficientes métodos de diversificación en su labor creativa y potencializará el éxito comercial, en torno a sus actividades profesionales. 


\section{Bibliografía}

Almela, R. (julio-agosto de 1998). Hacia el concepto del creador emergente. (M. Hernández Rosales, Ed.) Casa del Tiempo. Recuperado el 20 de noviembre de 2018, de http://www.uam.mx/difusion/revista/julio-agosto98/crea.html

Ayuntamiento de Guadalajara. (5 de octubre de 2006). Reglamento de Adquisiciones para el Municipio de Guadalajara. Guadalajara, Jalisco, México.

Ayuntamiento de Guadalajara. (1 de septiembre de 2018). 3er Informe de Gobierno, Ayuntamiento de Guadalajara. (A. d. Guadalajara, Ed.) Recuperado el 25 de noviembre de 2018, de https://portal.guadalajara.gob.mx/2018/pdf/Informe_2018_BAJAS.pdf

Aziz Nassif, A. (24 de julio de 2017). A 16 Años incumplen con el servicio civil de carrera. El Informador. Guadalajara, Jalisco, México. Recuperado de: https://www.informador.mx/Jalisco/A-16-anos-incumplen-con-el-serviciocivil-de-carrera-20170724-0127.html

Bossi, I. (enero de 2015). El método de negociación de la Universidad de Harvard: Ganar - Ganar. Revista Pymes. Recuperado el 25 de noviembre de 2018, de https://ucema.edu.ar/6/sites/default/files/2015/novedades/revista_pymes_0 12015.pdf

Carmona Medina, C. (1 de enero de 2015). Crear arte en México es vivir del estado. Milenio. Recuperado el 2018 de noviembre de 24, de http://www.milenio.com/cultura/crear-arte-en-mexico-es-vivir-del-estado

Corona, M. (25 de mayo de 2017). ¿Es posible vivir del arte (sin beca) en México? El Popular. Recuperado el 23 de Noviembre de 2018, de https://www.elpopular.mx/2017/05/25/opinion/es-posible-vivir-del-arte-sinbeca-en-mexico-163372

Gaceta Municipal del Ayuntamiento de Guadalajara. (14 de diciembre de 2017). Presupuesto de Egresos del Municipio de Guadalajara, para el ejercicio fiscal del año 2018. Guadalajara, Jalisco, México: Ayuntamiento de Guadalajara.

Gómez-Tagle, S. (30 de agosto de 2017). Mercadólogo enseña a creadores cómo vivir del arte. El Universal. Recuperado el 20 de noviembre de 2018, de 
http://www.eluniversal.com.mx/periodismo-de-investigacion/mercadologoensena-autores-como-vivir-del-arte

Hernández Sampieri, R., Fernández Collado, C. \& Baptista Lucio, P. (2014). Metología de la investigación (6ta. ed.). Ciudad de México, Álvaro Obregon, México: Mac Graw Hill.

LAV, CUAAD. (15 de abril de 2019). Perfil de egreso, Lic. Artes Visuales para la expresión plástica y expresión fotográfica. Obtenido de www.pregrado.udg.mx: http://www.pregrado.udg.mx/Centros/Tem\%C3\%A1ticos/CUAAD/artesvisuales-para-la-expresion-plastica-y-artes-visuales-para-la-expresionfotografica/perfil-egreso

Leal Jimenéz, A., \& Quero Gervilla, M. J. (2013). Manual de marketing y comunicación Cultural. Andalucía, España: Universidad de Cadiz y Universidad de Andalucía. Recuperado el 17 de mayo de 2019, de http://www.bizkaia.eus/home2/archivos/DPTO4/Temas/producto44manualde-marketing-y-comunicacioncultural_web.pdf?hash=8df797774ec44f13b1cf1efb9e0ddee5\&idioma=EU

Ley de Adquisiciones y Enajenaciones del Estado de Jalisco. (2 de julio de 2015). Decreto 25383/LX/15. Guadalajara, Jalisco, México: Diario Oficial del Estado de Jalisco.

Ley Federal del Derecho de Autor. (24 de diciembre de 1996). Ciudad de México, México: Diario Oficial de la Federación.

LIDA. (2018). Licenciatura en Diseño de Artesanías. (Universidad de Guadalajara) Recuperado el 21 de noviembre de 2018, de Portal de Programas Educativos de Pregrado, Universidad de Guadalajara: http://www.pregrado.udg.mx/Centros/Regionales/CUTONAL\%C3\%81/disenode-artesania/ambito-profesional

LIDA, Ámbito Profesional. (2018). Licenciatura en Diseño de Artesanía. Obtenido de Programas de Pregrado, Universidad de Guadalajara: http://www.pregrado.udg.mx/Centros/Regionales/CUTONAL\%C3\%81/disenode-artesania/ambito-profesional 
LIDA, Perfil de egreso. (2018). Licenciatura en Diseño de Artesanía. Obtenido de Universidad de Guadalajara: gestión, producción, comercialización y uso de productos

Meza Tejeda, E. (4 de enero de 2018). Artesanos de Jalisco registran marcas y constituyen empresas. El Economista. Recuperado el 2018 de noviembre de 25, de https://www.eleconomista.com.mx/estados/Artesanos-de-Jaliscoregistran-marcas-y-constituyen-empresas-20180104-0085.html

RAE. (25 de noviembre de 2018). Diccionario de la Lengua Española. Obtenido de Real Academia Española: http://dle.rae.es/?id=Oivh8dN

Ramírez, P. (20 de marzo de 2016). Esquemas de ingresos económicos para el sector creativo. Charla con responsables de gestión cultural en Jalisco. (Y. V. Mejía, Entrevistador). Radio UDG CUCIÉNEGA, Ocotlán.

Reglamento de la Administración Pública Municipal de Guadalajara. (27 de septiembre de 2018). Gaceta Municipal de Guadalajara. Guadalajara, Jalisco, México: Ayuntamiento de Guadalajara.

Secretaría de Cultura del Gobierno del Estado de Jalisco. (29 de octubre de 2018). Resultados: Proyecta Industrias Creativas 2018. Obtenido de Secretaría de Cultura del Gobierno de Estado de Jalisco: https://sc.jalisco.gob.mx/convocatorias/resultados-de-convocatorias-de-lasc/7841 